\title{
CONSULTA DE ENFERMAGEM EM GERIATRIA: TÓPICOS A SEREM ABORDADOS NO EXAME FÍSICO — PARTE II.
}

CAMPEDELLI, M. C. Consulta de enfermagem em geriatria: tópicos a serem abordados no exame físico - parte II. Rev. Esc. Enf. USP, v. 26, n. 1, p. 33-42, mar. 1992.

Neste trabalho são apresentados os itens considerados importantes para o exame físico do indivíduo idoso, além de outros tópicos que são fundamentais para a consulta de Enfermagem, complementando o artigo intitulado: "Consulta de Enfermagem em Geriatria: tópicos a serem abordados na Entrevista - parte I'.

UNITERMOS: Consulta de enfermagem. Enfermagem geriátrica.

\section{INTRODUÇĀO}

Em trabalho recente, apresentado no $2^{\circ}$ Encontro de Enfermagem em Tecnologia - ENFTEC, CAMPEDELLI et al ${ }^{8}$ especificaram o que deveria constar dos diversos itens propostos para a execução do exame físico do idoso, baseando-se nas principais alterações anátomo-fisiológicas que podem ser observadas nos indivíduos idosos.

Não entraremos em detalhes sobre o conceito, a importância e a operacionalização do exame físico, tendo em vista que há vários artigos abordando o assunto; entre eles podemos destacar: CRANE $^{10}$, GAIDZINSKI, KIMURA ${ }^{14}$, NAKAMAE, KOIZUMI ${ }^{24.25}$, SANA, JUDGE ${ }^{31}$ além de já os termos abordado no artigo apresentado no ENFTEC.

No entanto, acreditamos que haveria necessidade de apresentarmos algumas justificativas sobre os motivos de terem sido eleitos estes itens, propostos no modelo em anexo, baseando-se na nossa experiência profissional e na bibliografia específica quanto a esta faixa etária.

\section{DESENVOLVIMENTO DOS TÓPICOS A SEREM ABORDADOS}

Apresentaremos as justificativas seguindo a seqüência estipulada no modelo proposto por CAMPEDELLI'.

\footnotetext{
- Enfermeira. Professor Associado do Departamento de Enfermagem Médico-Cirúrgica da Escola de Enferma gem da USP.
} 
Sinais Vitais - Os sinais vitais são as chamadas informações básicas em qualquer atividade de atendimento na área da saúde.

A avaliação dos sinais vitais deve ser feita de forma cuidadosa, pois estes constituem elementos importantes para que a enfermeira possa estabelecer as suas condutas.

CUNHA $^{12}$ chama a atenção para a hipotensão ortostática que é extremamente comum na idade avançada, pois ela é causa importante de quedas.

$O$ peso e a altura são dados essenciais, em função principalmente da relação perda ou ganho de peso. ROSSMAN ${ }^{30}$ refere que as perdas de altura ocorrem na idade avançada e informa que, entre os 50 e 55 anos, já existe uma diminuição na ordem de 1 a 2 centímentros, que é atribuída ao encolhimento dos discos fibroelásticos entre as vértebras.

Quanto ao espaço designado para as observações foi previsto, por que a interpretação de sinais e sintomas, nos idosos, demanda reflexão cuidadosa, pois muitas vezes as alterações constituem manifestações genéricas de várias patologias. A enfermeira deve estar preparada tecnicamente para atuar na sua área de competência, detectando as alterações e indicando as condutas adequadas e preocupando-se em interagir com o paciente de forma efetiva e afetiva, já que ele fica nervoso ou muito tenso quando toma conhecimento de alteraçōes nos sinais vitais.

Condições de higiene - A avaliação das condições de higiene em todos os segmentos do corpo vai fornecer dados que refletem o grau de autocuidado, o nível de dependência e o sistema de apoio com o qual o idoso pode contar ${ }^{18}$.

"Aproveitar a vida e estar no meio social significa viver no meio dos outros, mostrando estar participando. Uma pessoa deixa de se autocuidar quando sente que a vida já não the oferece mais nada e está alí apenas para ver os dias passarem, perde a alegria, perde a crença nas coisas ..." ${ }^{29}$.

$\mathrm{Na}$ inspeção inicial, verificar as condições de higiene desde o cabelo, no sentido céfalo-caudal, até as unhas dos pés.

Cabeça e pescoço - Deverão ser anotados os problemas referentes às alterações em qualquer um destes segmentos, como no cabelo e couro cabeludo, pálpebras, nariz, boca e mucosa, língua, lábios, dentição, orelhas, face, garganta e pescoço.

A perda de dentes ou dentaduras adaptadas inadequadamente são responsáveis por vários problemas para os idosos ${ }^{13}{ }^{21}$.

A cefaléia de tensão é comum entre os idosos, pois os velhos são muito mais preocupados e tensos do que vulgarmente se difunde e o seu estade de ansiedade pode ser suficiente para mantê-los com persistente dor de cabeça, enfatiza AMÂNCIO'. Este autor ainda refere que a artrose cervical é virtualmente um acompanhante obrigatório do envelhecimento e pode entreter prolongados quadros de cefaléia e de parestesia, em geral de membros superiores.

Audição - GUIMARÃES ${ }^{19}$, ao abordar o problema da audição no idoso, descreve os vários tipos de sintomas, enfatizando que $30 \%$ das pessoas com 65 ou mais anos, apresentam diminuição de audição (Presbiacusia). Chama a atenção 
para os problemas psicossociais e acidentes que podem ser desencadeados, à medida que estes sintomas se agravam; refere ainda que o tinnitus* é apresentado por pelo menos um em cada dez idosos, ressaltando que é um sintoma muito importante em geriatria, pois ele causa um impacto negativo na qualidade de vida, o que é também enfatizado por PAIVA, MINITI ${ }^{27}$.

Como o aparelho auditivo está intimamente ligado à memória, à medida que se ouve mal a apreensão e a retenção se tornam mais difíceis, conforme enfatiza PACHECO e SILVA ${ }^{26}$. Para este mesmo este mesmo autor, o velho surdo sente isolamento muito grande, fica entregue a cogitação pessoais, é desconfiado e irrita-se facilmente, ao fazer interpretações das atitudes das pessoas com quem convive. Para compreender o que lhe foi dito, há necessidade de solicitar que o interlocutor repita as perguntas várias vezes; por isso sente que pode ser tomado por débil mental, incapaz de compreender certas coisas que, na realidade, não consegue ouvir.

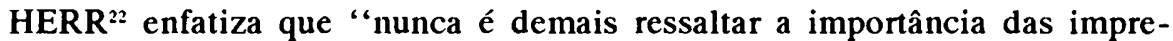
sões sensoriais para o bem estar psicológico', e COMFORT ${ }^{9}$ é enfático ao afirmar que a surdez interfere em todas as modalidades de interação social, principalmente nos dias de hoje, quando a sociedade não poupa esforços em isolar e se desfazer dos idosos.

Para o atendimento do idoso, há necessidade de pronunciar lenta e corretamente as palavras, pois assim estará sendo facilitada a compreensão.

Visão - MAGALHÃES ${ }^{23}$ refere que a pertubação da acuidade visual começa cedo (por volta dos 40 anos), como sinal de senilidade, e GIOILLA; BEVIT ${ }^{15}$ relatam que outro problema é a diminuiçãi do campo visual.

São cinco as principais causas de baixa da visão em idoso: presbiopia, catarata, degenereção da mácula, glaucoma e retinopatia diabética ${ }^{19}$.

PACHECO e SILVA ${ }^{26}$ chama a atenção da problemática causada pela pres-

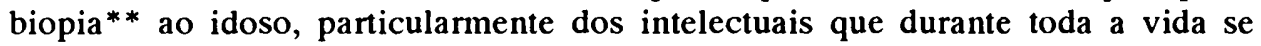
entregaram a constantes leituras.

Carvalho Filho ${ }^{28}$ ainda acrescenta que, ao atendermos o idoso, devemos estar atentos, pois sua memória começa a falhar concomitantemente com o enfraquecimento da visão.

Tronco anterior e posterior - Registrar os problemas referentes às alterações detectadas no tórax, abdômen e nas costas.

CUKIER et al." referem que o envelhecimento determina consideráveis modificações anatômicas no tórax, tanto radiologicamente quanto na medida do diâmetro transverso.

É importante que se aproveite o momento para orientar sobvre o auto-exame das mamas.

* tinnitus - audiçāo de ruídos desagradáveis e incômodos... zumbido ${ }^{19}$.

* Presbiopia ou vista cansada consiste na reduçāo da acuidade visual em conseqüência da dificuldade de acomodação do cristalino ${ }^{26}$ 
Membros - Geralmente existe uma atrofia lenta e constante dos músculos, que resulta em debilitação muscular. Com a perda da força muscular existe diminuição da força, resistência e agilidade. Gradativamente, os ossos perdem cálcio e se tornam mais porosos e mais leves. Os lígamentos calcificam-se e ossificamse, e as articulações tornam-se enrijecidas devido à erosões das superfícies articulares cartilaginosas. Como a falta de atividade agrava a incapacidade devido às alterações músculo-esqueléticas, os velhos necessitam ser encorajados a estarem fisicamente ativos dentro do nível de sua capacidade ${ }^{(3)}$.

Para GUIMARÃES et al. ${ }^{18}$, edema de membros inferiores e em particular "pés inchados" não são apanágio exclusivo de insuficiência cardíaca, mas também da falta de locomoção, varizes de membros inferiores e permanência excessiva em posição sentada. Chamam a atenção para que a temperatura e o aspecto das mãos, incluindo deformidades articulares, devam ser observados.

Medicações e tratamentos (prescritos e por conta própria) - Nas pessoas idosas, as polipatologias e a diversidade de sintomas apresentados faz com que elas se tornem grandes consumidoras de medicamentos. GUIMARĀES; GO$\mathrm{MES}^{20}$ ressaltam que nem sempre o idoso consegue seguir adequadamente o tratamento prescrito, devido a esquemas complexos e a um número variado de medicamentos que levam a erros involuntários.

Por outro lado, erros e complicaçōes podem advir na medida em que muitos idosos transgridem a terapêutica indicada, suprimindo algum medicamento ou mudando a sua dosagem, por acreditarem que tal produto não está fazendo bem, ou acrescentando outros, por ouvirem dizer que fez bem para um conhecido ou familiar.

BODACHNE ${ }^{2}$, no artigo em que aborda os principais acidentes entre os idosos, refere que esta categoria é mais vulnerável aos efeitos secundários dos medicamentos e que, com o processo de envelhecimento, ocorrem alterações orgânicas e funcionais que resultam no comprometimento da absorção, distribuição, metabolismo e excreção dos medicamentos.

Há necessidade de que a enfermeira, na Consulta de Enfermagem, investigue cuidadosamente este aspecto, pois assim poderá evitar danos maiores e orientar adequadamente o paciente.

Qual é a maior preocupação no momento - O objetivo é dar oportunidade para que o cliente fale a respeito de suas preocupações sob todos os pontos de vista (físico, social, econômico, psicológico e espiritual).

Os dados obtidos devem ser analisados criteriosamente e, se possível, confrontados com os fornecidos pelos familiares ${ }^{32}$.

GIOILLA, BEVIT ${ }^{16}$ chamam a atenção para o receio do idoso de que seus relatos sejam subestimados, principalmente quando ele sente que está tomando muito tempo da enfermeira e ela se mostra muito ocupada.

Para SKEET ${ }^{33}$, "uma das necessidades mais comuns dos idosos é a assistência; não é suficiente que cuidemos deles mas que lhes dispensemos assistência material e carinho; carinho e assistência material que lhes proporcionem conforto e apoio em momentos de ansiedade, solidão e desamparo. Tal assistência inclui 
dar ouvido a seus anseios e necessidades e, posteriormente, intervir de forma adequada e positiva, esta atitude é o elemento básico da enfermagem em todos os países do mundo".

RONCARATTI ${ }^{29}$, em uma das considerações finais da sua pesquisa, cujo objetivo foi "conhecer a relação, juntamente com os membros da equipe de emfermagem, entre os cuidados prestados aos pacientes e a aceitação da velhice e do envelhecer" afirmou que "trabalhar a velhice no ambiente hospitalar foi uma grande lição de viver, de ouvir e refletir...".

A enfermeira deve mostrar disponibilidade para ouvir e competência para intervir e auxiliar no momento adequado.

Observações - É de suma importância que a enfermeira esteja alerta para o registro das observações, pois, embora possa parecer que a assistência de enfermagem em essência não seja diferente para as pessoas idosas, o conhecimento das características inerentes a essa fase do ciclo vital impõe aos profissionais de enfermagem uma atenção especial para intervir de forma adequada e positi$v^{4}{ }^{4}$ o que também foi enfatizado por GUIMARÃES ${ }^{17}$; este afirmou que a " a interpretação de observações realizadas em torno de um paciente idoso, requer do enfermeiro o conhecimento das alteraçōes decorrentes de seu estado, a fim de não fundamentar os cuidados de enfermagem em afirmações incompletas e estereotipadas".

A enfermeira deve estar atenta para observar e orientar se o cliente apresenta problema em relação a sua auto-imagem, pois "na nossa sociedade é valorizado o novo e o produtivo, o que faz com que muitos velhos, com raras exceções, considerem-se feios, inúteis, e fiquem esperando a morte, isolando-se cada vez mais na frente de uma televisão, esperando o tempo passar'".

Cabe ressaltar que aquilo que se observa, pode muitas vezes ter um significado mais importante do que aquilo que o paciente relatou, e isto vai repercurtir na qualidade e adequação do cuidado.

Condutas - As condutas são os registros das intervenções de enfermagem, que CAMPEDELLI' estabeleceu como:

- prestar os cuidados que se fizerem necessários;

- ministrar as orientações indicadas no momento e

- encaminhar para outros profissionais quando a competência de resolução do problema fugir do seu âmbito de ação.

É importante que as enfermeiras valorizem o registro das condutas; se paralelamente chegarem a um vocabulário próprio e uniforme, facilitarão a comunicação entre elas próprias e com os outros profissionais da área da saúde. Como conseqüência, o idoso terá melhor a assistência de enfermagem e, com certeza, a consulta de enfermagem propiciará a resoluçāo de maior número de problemas.

Anotar a data, assinar após o término da consulta no espaço reservado para a assinatura da enfermeira, e registrar o número do Conselho Regional de Enfermagem - COREN $-n^{2}$.

\section{CONCLUSĀO}

Os itens propostos para o exame físico, na consulta de enfermagem, pos- 
sibilitam levantar os problemas mais comuns do idoso, nos ambulatórios, pois permitem detectar as alterações mais frequientes que ocorrem nesta faixa etária.

Pretendemos, com esta apresentação, que as enfermeiras interessadas sobre este assunto testem o instrumento e façam as adaptações necessárias, a fim de que, de fato, possamos atender ao idoso de maneira mais efetiva, e resolver a maior parte de seus problemas, contribuindo, assim, para melhoria de sua qualidade de vida.

CAMPEDELLI, M.C. Nurses consultation on geriatrics: justificatión for physical exam - part II. Rev. Esc. Enf. USP., v. 26, n. 1, p. 33-42, mar. 1992.

The author approachers physycal exam in elderly and other items that are essential to nurses consultation. This article completes the topic Nurses consultation on Geriatrics - part I.

UNITERMOS: Nurses consultation Geriatric nursing.

\section{REFERENCIAS BIBLIOGRÁFICAS}

01. AMÂNCIO, A. Problemas neurológicos na velhice. In: AMÂNCIO, A.; CAVALCANTI, P.C.U. Clínica geriátrica. Rio de Janeiro, Atheneu, 1975. p. 57-71.

02. BODACHNE, L. Acidentes entre os idosos. Geriatr. Síntese, v. 4, n. 1, p. 9-12, 1987.

03. BRUNNER, L.S.; SUDDARTH, H.D.S. Enfermagem médico cirúrgica. 4. ed. Rio de Janeiro, Interamericana, 1982. p. 209-35: Apoio de enfermagem à pessoas idosas.

04. CAMPEDELli, M.C. Atuação de enfermagem em geriatria e gerontologia. Rev. Paul. Hosp., v. 31, n. $9 / 10$, p. $198-200,1983$.

05.

Consulta de enfermagem ao binômio mãe-filho: análise das condutas de enfermagem. Sāo Paulo, 1986. 118p. Tese (Doutorado) - Faculdade de Saúde Pública, Universidade de Sāo Paulo.

06. Temos medo de envelhecer? editorial. Bol. Inf. ENC, v. 2, n .5, p.1, 1989.

07.

Consulta de enfermagem em geriatria: proposta de modelo. Sāo Paulo, 1989, 92p. Tese (Livre-Docência) - Escola de Enfermagem, Universidade de São Paulo.

08. CAMPEDELLI, M.C. et al. Enfermagem em geriatria: subsídios para um exame fisico. Apresentado no $2^{2}$ Encontro de Enfermagem em Tecnologia - ENFTEC, Sāo Paulo, 1990. 13p.

09. COMFORT, A. A boa idade. São Paulo, Difel, 1979.

10. CRANE, J. El examen fisico: un aspecto en la evoluacion clinica de enfermeira. In SANA, J.M.; JUDGE, R.D. Métodos para el examen físico en la prática de enfermeria. Cali, Organización Panamericana de la Salud, 1977. cap. 2, p. 11-26.

11. CUKIER, A. et al. Afecçōes pulmonares. In: SERRO AZUL, L.G.C.C. de et al. Clínica do indivíduo idoso. Rio de Janeiro, Guanabara Koogan, 1981. p.51-6.

12. CUNHA, U.Q. de V. Os grandes problemas geriátricos. Geriatr. Síntese, v.2, n. 5, p. 17-20, 1985.

13. CUNHA, U.Q de V. Sinais e sintomas inespecíficos. In: GUIMARĀES, R. M.; CUNHA, U.G. de V. Sinais e sintomas em geriatria. Rio de Janeiro, Revinter, 1989. p. 19-27. 
14. GAIDZINSKI, R.R.; KIMURA, M. Entrevista e exame físico-instrumentos para levantamento de dados. In: CAMPEDELLI. M.C. et al. Processo de enfermagem na prática. Sāo Paulo, Ática, 1989. p. 66-8.

15. GIOLLA, E.C.; BEVIT. C.W. Nursing care of the aging client: promoting healthy adaptation. Norwalk, Appleton Century Crofts, 1985. p. 154-63: Preparing the client for healthy aging.

16. Nursing care of the aging client: promoting healthy adaptation. Norwalk, Appleton Century Crofts, 1985. p. 189-96: Identifying the client at risk.

17. GUIMARĀES, R.M.M. Assistência à um paciente geriátrico - estudo de caso. Rev. Bras. Enf., v. 31, n. 4 , p. $542-66,1978$.

18. GUIMARĀES, R.M. et al. Avaliaçāo do paciente geriátrico. de V. Sinais e sintomas em geriatria. Rio de Janeiro, Revinter, 1989. p. 7-17.

19. GUIMARĀES, R.M. Distúrbios visuais e auditivos. In: Sinais e sintomas em geriatria. Rio de Janeiro, Revinter, 1989. p. 107-11.

20. GUIMARĀES, R.M.; GOMES, F.A. Reaçōes adversas a drogas. In: ; CUNHA, U.G. de V. U.G. de V. Sinais e sintomas em geriatria. Rio de Janeiro, Revinter, 1989. p. 181-6.

21. GUNTER, L.M.; MILLER, J.C. Toward a nursing gerontology Nurs. Res., v. 26, n. 3. p. 208-21, 1977.

22. HERR, J.J. Psicologia do envelhecimento: uma visāo geral. gem e os idosos. Sāo Paulo, Organizaçāo Andrei, 1979. p. 26-33.

23. MAGALHĀES, P.B. de. Afecçōes oftalmológicas. In: SERRO AZUL, L.G.C.C. de et al. Clínica do indivíduo idoso. Rio de Janeiro, Guanabara Koogan, 1981. cap. 5, p. 44-5.

24. NAKAMAE, D.D.; KOIZUMBI, M.S. Exame clínico de enfermagem: condiçōes físicas (1' parte). Enf. Novas Dimens., v. 4, n. 6, p. 342-5, 1978.

25. Exame clínico de enfermagem: condiçōes físicas (2' parte). Enf. Novas Dimens., v. 5, n. 1, p. 10-22, 1979.

26. PACHECO e SILVA, A.C. O problema da sensibilidade do mundo moderno. In: SERRO AZUL, L.G.C.C. de et al. Clínica do indivídùo idoso. Rio de Janeiro, Guanabara Koogan, 1981. p. 341-5.

27. PAIVA, L.J.; MINITI, A. Afecçōes otorrinolaringológicas. In: SERRO AZUL, L.G.C.C. de al. Clínica do indivíduo idoso. Rio de Janeiro, Guanabara Koogan, 1981. p. 46-50.

28. QUANDO nossa máquina começa a falhar. Estado de São Paulo., São Paulo, 27 ago. 1989. Suplemento Feminino, p. 14.

29. RONCARATTI, E. Trabalhando a velhice no ambiente hospitalar. Sāo Paulo, 1988. Monografia (Curso de Especializaçāo em Enfermagem Psiquiátrica e Saúde Mental) - Departamento de Enfermagem, Escola Paulista de Medicina.

30. ROSSMAN, I. Alterações humanas no envelhecimento. In: BURNSIDE, I.M. Enfermagem e os idosos. São Paulo, Organizaçāo Andrei, 1979. p. 68-78.

31. SANA,J.M.; JUDGE ,R.D. Métodos para el examen físico en la práctica de enfermeria. Cali, Organizacion Panamerica de la Salud, 1977.

32. SERRO AZUL, L.G.C.C. de el al. O primeiro contato com o paciente. In: SERRO AZUL, L.G.C.C. de el al. Clínica do indivíduo idoso. Rio de Janeiro, Guanabara, Koogan, 1981. p. 12-22.

33. SKEET,M. A valorizaçăo da velhice: o papel da enfermagem. In: ORGANIZAÇĀO MUNDIAL DA SAÚDE. Remoçar a velhice. Geneva, 1982. p. 1-3 (Dia Mundial da Saúde, 4). 
CONSULTA DE ENFERMAGEM

Geriatria

Nome:

RG:

Sexo:

Idade:

Estado Civil:

Há quanto tempo:

Religião:

Instrução:

Ocupação anterior:

Ocupação atual:

Onde mora:

Com quem mora:

Porque procurou o hospital:

Quem responde?: idoso ( ) outro ( ) especificar

Características da habitação

Lazer e recreação

Sono e repouso

Locomoção, atividades físicas e esforços

Alimentação e hidratação 
Eliminações: urinária

fecal

Tabagismo e etilismo

Sexualidade

Condições de comunicação

Condições de orientação no tempo e espaço

Outras informaçōes

Sinais Vitais: $\mathbf{T}$ :

$\mathbf{P}$

$\mathbf{R}$

PA

Peso:

Altura Observações

Condições de higiene

Cabeça e pescoço

Audição 
Visão

Tronco anterior e posterior

Membros

Medicações e tratamentos - (prescritos; e por conta própria)

Qual é a sua maior preocupação no momento?

Observações

Condutas

Data Assinatura da enfermeira 